AC.

$$
\begin{aligned}
& \text { KEK P 93-185 } \\
& \text { Sw 9412 }
\end{aligned}
$$

KEK Preprint 93-185

January 1994

$\mathbf{H} / \mathbf{M}$

\title{
T-violation in Neutron Spin Rotation
}

Yasuhiro MASUDA

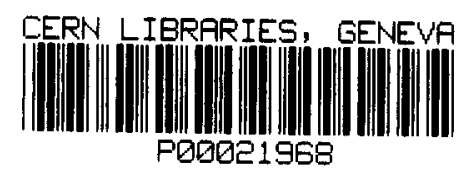

To be published. 
National Laboratory for High Energy Physics, 1994

KEK Reports are available from:

Technical Information \& Library

National Laboratory for High Energy Physics

1-1 Oho, Tsukuba-shi

Ibaraki-ken, 305

JAPAN

Phone: $0298-64-1171$

Telex: $\quad 3652-534$ (Domestic)

(0)3652-534 (International)

Fax: $\quad 0298-64-4604$

Cable: KEK OHO

E-mail: LIBRARY@JPNKEKVX (Bitnet Address)

library@kekvax.kek.jp (Internet Address) 


\title{
$T$-violation in Neutron Spin Rotation
}

\author{
Yasuhiro Masuda \\ National Laboratory for High Energy Physics \\ 1-1 Oho, Tsukuba-shi, Ibaraki-ken, 305 Japan
}

\begin{abstract}
A new $T$-violation experiment concerning the neutron-nucleus interaction is proposed. In this experiment the neutron spin rotates around the nuclear spin by $180^{\circ}$ from the forward to backward direction during propagation through the target. The rotation direction and incident neutron spin are reversed at a suitable period. The $T$-violation effect is found in the asymmetry of the transition probability of the neutron spin state with respect to the two kinds of reversals. The systematic error can be small compared with the $T$-violation limit bound by the neutron EDM measurement.
\end{abstract}

\section{Introduction}

$T$-violation in the neutron-induced reaction in the $\mathrm{eV}$ region is one of the recent topics concerning fundamental symmetry violations, since a large enhancement of the $P$-odd $\sigma \cdot k$ correlation term has been found in the $p$-wave resonance of the neutron-radiative-capture reaction.[1, 2, 3, 4] $\sigma$ is the neutron spin and $k$ the neutron momentum. Several kinds of $T$ violation experiments have been proposed. Among them, a measurement of the $T$-odd and $P$-odd $\sigma \cdot(\boldsymbol{k} \times \boldsymbol{I})$ correlation term, where $\boldsymbol{I}$ is the nuclear spin, is most interesting, since it has the following three advantages.

Firstly, a large enhancement is expected. Secondly, the effect is free from final-state interactions, since the initial and final states are the same plane wave. Thirdly, the ratio of the $T$-odd to $P$-odd effect is insensitive to the nuclear wave-function uncertainty. As a result, the measurement can be compared with other elementary-particle experiments, for example the neutron electric-dipole-moment (EDM) measurement. Therefore, many experimental methods have been proposed to measure the $T$-odd and $P$-odd term.

At first, neutron-spin rotation around $k \times \boldsymbol{I}$ during propagation through a polarized nuclear target was proposed.[5] However, fake effects due to the coupling of two different kinds of spin-correlation terms, $\sigma \cdot \boldsymbol{k}$ and $\sigma \cdot \boldsymbol{I}$, disable one from carrying out an accurate measurement. 
Measuring the transmission asymmetry and polarization for the neutron spin in the $k \times I$ axis was also proposed.[6] According to the polarizationasymmetry theorem, fake effects are canceled out in the sum of the polarization and asymmetry.[7] A detailed balance in spin-flip processes induced by the $\sigma \cdot(\boldsymbol{k} \times \boldsymbol{I})$ correlation term is a good candidate to obtain the $T$-violation effect without any fake effects, since the two processes, which are time-reversed processes for each other, are compared in its measurement.[6] In these measurements, however, it is still very difficult to obtain $T$-violation information beyond the present limit bound by the neutron EDM measurement.

Here, we discuss a new method, which breaks through the problem. The present method involves a double asymmetry in the transition probability of neutron spin states during propagation through a polarized nuclear target. During propagation, the neutron spin rotates by $180^{\circ}$ around the nuclear spin from forward to backward and vice versa. The $T$ violation effect is found in the difference in the transition probability upon a reversal of the rotation as well as upon a reversal of the incident neutron spin. The present method is a combination of the $T$-odd transmission asymmetry and the spin-detailed balance. The size of the present $T$-violation effect is as large as the $T$-odd transmission asymmetry and polarization. There is no fake effect in the present asymmetry like that in the spin detailed balance. Furthermore, another systematic error which may be induced by a mis-alignment between the neutron spin and nuclear spin is canceled in the double asymmetry. As a result, a considerable improvement is expected in the $T$-violation limit.

II. Rotation asymmetry as a measure of the $T$-violation

The propagation of a polarized neutron through a polarized nuclear target is discussed in terms of the forward scattering amplitude $(f(0))$ and the density matrix.[8] The general form of the scattering amplitude for the polarized nucleus can be described as

$$
\begin{aligned}
f(0) & =F_{0}+F_{1} \sigma_{x}+F_{2} \sigma_{y} \quad+F_{3} \sigma_{z} \\
& =F_{0}+F_{1} \sigma \cdot \boldsymbol{I}+F_{2} \sigma \cdot(\boldsymbol{k} \times \boldsymbol{I})+F_{3} \sigma \cdot \boldsymbol{k} .
\end{aligned}
$$

Here, $\sigma$ is the Pauli spin operator. [6] $F_{i}(i=0,1,2,3)$ are scattering amplitudes. After propagation through the target, the effect of the nuclear interaction is found in a phase shift $(\Delta)$ of the neutron wave,

$$
\Delta=\lambda \operatorname{lNf}(0)
$$

Here, $\lambda$ is the neutron wavelength, $l$ the neutron propagation-length and $N$ the target-nucleus-number density. The density matrices of the incident 
neutron spin states, which are parallel and anti-parallel to the neutron momentum ( $z$ axis), are

$$
\rho_{ \pm}=1 / 2 \cdot\left(1 \pm \sigma_{z}\right)
$$

respectively. After propagation, the density matrices are modified in the presence of the phase shift as

$$
\rho_{\mathrm{f}}=F \rho_{ \pm} F \dagger
$$

where, $F$ is

$$
F=\exp (\mathrm{i} \Delta)
$$

The $180^{\circ}$ neutron spin rotation is described in terms of the density matrices and projection operators $\left(P_{ \pm}\right)$for parallel and anti-parallel spin states to the $z$-axis

$$
P_{ \pm}=\left(1 \pm \sigma_{z}\right) / 2
$$

The transitions from positive to negative and from negative to positive state ( $R_{+-}$and $R_{-+}$) are described as follows:

$$
\begin{aligned}
R_{+-}= & \operatorname{Tr}\left(F \rho_{+} F \dagger P_{-}\right) \\
= & \exp \left(-2 \operatorname{Im}\left(\phi_{0}\right)\right)(\sin b / b)^{2} \\
& \left\{\left|\phi_{1}\right|^{2}\left|\phi_{2}\right|^{2}+2\left(\operatorname{Im}\left(\phi_{1}\right) \cdot \operatorname{Re}\left(\phi_{2}\right)-\operatorname{Re}\left(\phi_{1}\right) \cdot \operatorname{Im}\left(\phi_{2}\right)\right\},\right.
\end{aligned}
$$

and

$$
\begin{aligned}
R_{-+}= & \operatorname{Tr}\left(F \rho_{-} F \dagger P_{+}\right) \\
= & \exp \left(-2 \operatorname{Im}\left(\phi_{0}\right)\right)(\sin b / b)^{2} \\
& \left\{\left|\phi_{1}\right|{ }^{2}\left|\phi_{2}\right|^{2}-2\left(\operatorname{Im}\left(\phi_{1}\right) \cdot \operatorname{Re}\left(\phi_{2}\right)-\operatorname{Re}\left(\phi_{1}\right) \cdot \operatorname{Im}\left(\phi_{2}\right)\right\},\right.
\end{aligned}
$$

respectively. Here,

$$
b=\sqrt{\left|\phi_{1}\right|^{2}+\left|\phi_{2}\right|^{2}+\left.\phi_{3}\right|^{2}}
$$

and $\phi_{i}$ are spin-dependent phase shifts, which can be represented by

$$
\phi_{i}=\lambda l N F_{i} \quad(i=0,1,2,3) .
$$

In the eq. (9), $\left|\phi_{1}\right|^{2}$ is dominant, 


$$
b^{2}=\left|\phi_{1}\right| 2\left(1+\delta_{\phi}\right)
$$

In the p-wave resonance of ${ }^{139} \mathrm{La}$,

$$
\operatorname{Re}\left(\phi_{1}\right) » \operatorname{Im}\left(\phi_{1}\right) \text {. }
$$

The dominant term in $R_{+-}$and $R_{+}$is $\left|\operatorname{Re} \phi_{1}\right|^{2}$. According to the theoretical prediction ${ }^{[9]}$ and a recent experiment concerning parity-violating neutron-spin rotation, $[10]$

$$
\operatorname{Im}\left(\phi_{3}\right) \gg \operatorname{Re}\left(\phi_{3}\right)
$$

As a result,

$$
R_{+-}=\exp \left(-2 \operatorname{Im}\left(\phi_{0}\right)\right)(\sin b)^{2}\left(1-\delta_{\phi}\right)\left\{1-2 \operatorname{Im}\left(\phi_{2}\right) / \operatorname{Re}\left(\phi_{1}\right)\right\},
$$

and

$$
R_{-+}=\exp \left(-2 \operatorname{Im}\left(\phi_{0}\right)\right)(\sin b)^{2}\left(1-\delta_{\phi}\right)\left\{1+2 \operatorname{Im}\left(\phi_{2}\right) / \operatorname{Re}\left(\phi_{1}\right)\right\} .
$$

Since the neutron spin rotates around the nuclear polarization ( $x$ axis) by $180^{\circ}$ from forward to backward and vice versa, the neutron spin is in $+y(k \times I)$ region and $-y$ region during the propagation through the target, respectively. The imaginary part of the phase shift $\left(\phi_{2}\right)$, which is the $T$-odd term, is found in the neutron wave after propagation. The sign of the $T$-odd term is changed upon reversal of the incident neutron spin. From this point of view, the present method is similar to the $T$-odd transmission asymmetry. The asymmetry of the transition probabilities ( $R_{+-}$and $R_{-+}$) is

$$
\begin{aligned}
A_{\mathrm{S}} & =\left(R_{+-}-R_{-+}\right) /\left(R_{+-}+R_{-+}\right) \\
& =-2 \operatorname{Im}\left(\phi_{2}\right) / \operatorname{Re}\left(\phi_{1}\right) .
\end{aligned}
$$

Eq. (16) is essentially the same as the spin-detailed balance. If the value of $\lambda l N$ is

$$
\lambda l N=2.4 \times 10^{14} \mathrm{~cm}^{-1},
$$

the value of $A_{\mathrm{S}}$ is

$$
A_{\mathrm{S}}=1.4 \times 10^{-2} \eta P_{\mathrm{I}} \text {. }
$$

Here, $\eta$ is 


$$
\eta=\operatorname{Im}\left(\phi_{2}\right) / \operatorname{Im}\left(\phi_{3}\right)
$$

The sign of the $T$-odd term is also changed upon a rotation reversal. The asymmetry of the transition probability with respect to the rotation reversal, which is referred to as rotation asymmetry, is

$$
\begin{aligned}
A_{\mathrm{r}}(+) & =\left(R_{+-}\left(+180^{\circ}\right)-R_{+-}\left(-180^{\circ}\right)\right) /\left(R_{+-}\left(+180^{\circ}\right)+R_{+-}\left(-180^{\circ}\right)\right) \\
& =-2 \operatorname{Im}\left(\phi_{2}\right) /\left|\operatorname{Re} \phi_{1}\right|,
\end{aligned}
$$

when the incident spin is in the $+z$ direction. When the incident spin is in the $-z$ direction,

$$
\begin{aligned}
A_{\mathrm{r}}(-) & =\left(R_{+}\left(+180^{\circ}\right)-R_{-+}\left(-180^{\circ}\right)\right) /\left(R_{-+}\left(+180^{\circ}\right)+R_{-+}\left(-180^{\circ}\right)\right) \\
& =+2 \operatorname{Im}\left(\phi_{2}\right) /\left|\operatorname{Re} \phi_{1}\right| .
\end{aligned}
$$

III Experimental scheme and systematic error

The experimental scheme is shown in Fig. 1. The neutron beam is polarized by either a polarized ${ }^{3} \mathrm{He}$ filter or a polarized proton filter. A neutron enters into a target section through a flipper section. After propagation through the target, the neutron spin is analyzed by a ${ }^{3} \mathrm{He}$ neutron polarimeter.[11] The neutron spin is manipulated as follows.

After transmission through the polarizer, the neutron spin rotates following an adiabatic passage upstream of the flipper. The magnetic field direction is along the $y$-axis at the flipper and reversed at a suitable period. The neutron spin is reversed with a reversal of the field direction. After the flipping magnet, the neutron spin enters into a $90^{\circ}$ coil through a superconducting sheet. In the superconductor the magnetic field is zero due to the Miesner effect. The neutron sees a sudden decrease in the magnetic field to zero upon passing through the surface of the superconductor. The magnetic field penetration-depth of niobium is $\lambda(0)$ $=440 \AA$ at $\mathrm{T}=0 \mathrm{~K}$. The neutron time of flight for the penetration depth is $3.7 \times 10^{-12} \mathrm{sec}$ at a neutron energy of $E_{\mathrm{n}}=0.734 \mathrm{eV}$. The neutron spin does not follow this sudden change in the magnetic field, because the rate of the magnetic field change is very fast compared with the Larmor frequency, which is $2.9 \times 10^{3} \mathrm{~Hz} / \mathrm{G}$. As a result, the neutron spin enters into the $90^{\circ}$ coil without any change in its direction. In the $90^{\circ}$ coil, the magnetic field direction is along the $x$-axis. The neutron spin rotates from the $y$ - to $z$ - direction before entering into the polarized nuclear target. In the target section, a static magnetic field $\left(H_{0}\right)$ is applied in order to hold the nuclear polarization. In addition to the magnetic field, the neutron sees a field called a "pseudomagnetic field $\left(H^{*}\right)$ " due to the $F_{1}$ term during transmission through the target. Therefore, the neutron-spin 
rotates in the sum of these fields $\left(H_{0}+H^{*}\right)$. The neutron-spin-rotation is controlled by adjusting the magnetic field $\left(\mathrm{H}_{0}\right)$ so that neutron-spinrotation angle is either $180^{\circ}$ or $-180^{\circ}$ during transmission. After transmission, the neutron spin rotates from the $z$-to $y$ - direction in the second $90^{\circ}$ coil and is then analyzed by a polarized ${ }^{3} \mathrm{He}$ neutron polarimeter.

In the neutron-spin manipulation, detection of the neutron-spinrotation angle is very important. We have developed a rotation phase detector by using the Meisner effect.[12] The neutron-spin-rotation phase is converted to a projection angle on the magnetic field. The projection component of the neutron spin is measured by the ${ }^{3} \mathrm{He}$ neutron polarimeter. In this method, an accuracy of $\delta \theta<1^{\circ}$ has been found to be possible. If we use a $\mathrm{LaF}_{3}$ or $\mathrm{LaAlO}_{3}$ single crystal as a polarized nuclear target $[13,14]$ and the nuclear polarization is $50 \%$, the pseudomagnetic field is $\sim 1 \mathrm{kG}$. Neutrons of $E_{\mathrm{n}}=0.734 \mathrm{eV}$ rotate $\sim 10$ turns upon passage through a $1 \mathrm{kG}$ magnetic field for a $4-\mathrm{cm}$ length. A $1^{\mathrm{O}}$-rotation adjustment means a field adjustment of $\delta \mathrm{H}_{\mathrm{o}} / \mathrm{H}_{\mathrm{o}}=3 \times 10^{-4}$.

If we assume that the uncertainty of the neutron-spin rotation is $\delta \theta$, the uncertainty of the phase of the neutron wave $(\delta)$ is

$$
\delta=\delta \theta / 2 .
$$

Then, the real part of $\phi_{1}$ is

$$
\operatorname{Re}\left(\phi_{1}\right)=\pi / 2+\delta
$$

In the measurement of $A_{\mathrm{S}}$, the effect of the rotation uncertainty is canceled, if the common coefficient, $\exp \left(-2 \operatorname{Im}\left(\phi_{0}\right)\right)(\sin b)^{2}\left(1-\delta_{\phi}\right)$, is same under neutron-spin flipping. The values of $\exp \left(-2 \operatorname{Im}\left(\phi_{0}\right)\right)$ and $(1-$ $\left.\delta_{\phi}\right)$ are assumed to be constant. The value of $(\sin b)^{2}$ may change during neutron spin flipping, since it has a small polarization dependence,

$$
b=\pi / 2+\delta+r_{1} / 2+\delta_{\phi} / 2 .
$$

Here,

$$
r_{1}=\left(\operatorname{Im}\left(\phi_{1}\right) / \operatorname{Re}\left(\phi_{1}\right)\right)^{2}<0.01 P_{\mathrm{I}^{2}}^{2} \quad\left(\text { for } \operatorname{Re}\left(\phi_{1}\right)=\pi / 2\right) .
$$

Here, $P_{\mathrm{I}}$ is the nuclear polarization. As a result,

$$
\begin{gathered}
(\sin b)^{2}=\left(\cos \left(\delta+r_{1} / 2+\delta_{\phi}\right)\right)^{2} \\
=1-\left(\delta+r_{1} / 2+\delta_{\phi}\right)^{2} .
\end{gathered}
$$


If the change in $\delta$ upon neutron-spin flipping is $0.01^{\circ}$, the change in ( $\sin b$ )$^{2}$ is

$$
\Delta\left((\sin b)^{2}\right)<4 \times 10^{-6} .
$$

This change corresponds to an error in the ratio $\eta$ of

$$
\delta \eta<3 \times 10^{-4}
$$

for a nuclear polarization of 50\%. If we assume a relaxation time of $10 \mathrm{~h}$ for nuclear polarization and a flipping period of $4 \mathrm{sec}$, the change in $(\sin b$ )$^{2}$ is

$$
\Delta\left((\sin b)^{2}\right)<1 \times 10^{-6} .
$$

In addition to the above-mentioned errors, a systematic error may be induced due to any mis-alignment between the neutron polarization and nuclear polarization. If the neutron-polarization direction slightly deviates by $\delta_{\mathrm{n}}$ from $90^{\circ}$ with respect to the nuclear polarization, a $\sin \delta_{\mathrm{n}}$ component of the neutron polarization is held in the direction of the nuclear polarization during transmission through the target. This misalignment induces spin-dependent absorption which is proportional to $\sin \delta_{\mathrm{n}} \operatorname{Im}\left(\phi_{1}\right)$. Since the sign of $\delta_{\mathrm{n}}$ is reversed upon neutron-spin flipping, the effect of the mis-alignment remains in the value of $A_{\mathrm{S}}$. However, the absorption effect is small and independent of the neutron-spin-rotation direction. Therefore, the mis-alignment effect is canceled in the rotation asymmetry $\left(A_{\mathrm{r}}\right)$. As a result, the systematic errors are greatly reduced if we take a double asymmetry $\left(A_{d}\right)$ of

$$
A_{\mathrm{d}}=\left(A_{\mathrm{r}}(+)-A_{\mathrm{r}}(-)\right) /\left(A_{\mathrm{r}}(+)+A_{\mathrm{r}}(-)\right) .
$$

We can expect a large $T$-violation effect in the $T$-odd transmission asymmetry and polarization. However, it is very difficult to reduce the fake effect in these measurements. Although the fake effect is canceled in the sum of the $T$-odd transmission asymmetry and polarization, [7] the two measurements are independent of each other; therefore, the fake effect dose not necessarily have the same value in the two measurements. In the spin-detailed balance there is no fake effect, since any difference in spin-flip probabilities which are time reversed to each other is measured.[6] Cancellation of the Larmor and pseudomagnetic precessions makes the effect of the $F_{1}$ term on the spin flip small, so that the effect of the $F_{2}$ term increases in the spin-detailed balance. However, the accuracy is limited, because we should measure any small difference between small spin-flip probabilities.[15] Also, any mis-alignment between the neutron spin and the nuclear polarization induces a spurious effect. Based on this 
point of view, the present method has great advantage. The value of $A_{\mathrm{s}}$ or $A_{\mathrm{r}}$ is as large as the $T$-odd transmission asymmetry and polarization. There is no fake effect as in the spin-detailed balance. The mis-alignment effect is also canceled. As a result, a comparison with the neutron EDM measurement is possible.

The present experimental accuracy of the neuron EDM corresponds to a $\eta$ value of

$$
\eta<4 \times 10^{-3} \quad \text { (n EDM) }[8,16]
$$

Therefore, the rotation asymmetry has a possibility to yield more accurate information than the neutron EDM.

The author would like to thank Dr. V.P. Gudkov, Prof. K. Asahi and J.D. Bowman for their valuable discussions. He also expresses his thanks to his colleague concerning $P$-violation and $T$-violation experiments carried out at KEK for their indispensable collaboration. He also thanks Prof. H. Sugawara, Prof. N. Watanabe and Prof. K. Nakai for their warm encouragement.

[1] V.P. Alfimenkov et al., Nucl. Phys. A398(1983)93.

[2] Y. Masuda et al., Hyp. Int. 34(1987) 143; Nucl. Phys. A478(1988)737c; Nucl. Phys. A504(1989)269.

[3] S.A. Biryukov et al., Sov. J. Nucl. Phys. 45(1987)937.

[4] C.D. Bowman et al., Phys. Rev. C39(1989)1721.

[5] V.E. Bunakov and V.P. Gudkov, Z. Phys. A308(1982)363.

[6] L. Stodolsky, Physics Lett. B172(1986)5.

[7] P.K. Kabir, Phys. Rev. Lett. 60(1988)686.

[8] I.I. Grevich and L.V. Tarasov, "Low-Energy Neutron Physics", North Holland (1968).

[9] V.P. Gudkov, Phys. Rep. 212(1992)77.

[10] K. Sakai et al., to be published in Hyp. Int.

[11] H. Sato et al., to be published in Hyp. Int.

[12] Y. Masuda, Neutron Research 1(1993)53.

[13] Y. Masuda et al., KEK report 90-25 (1991) 1002.

[14] Y. Masuda et al., Proc. 18th INS Int. Symp., World Scientific(1991)293.

[15] Y. Masuda et al., Hyp. Int. 74(1992)149.

[16] P. Herczeg, Hyp. Int. 75(1992)127.

Fig. 1 Experimental scheme of the rotation asymmetry. 


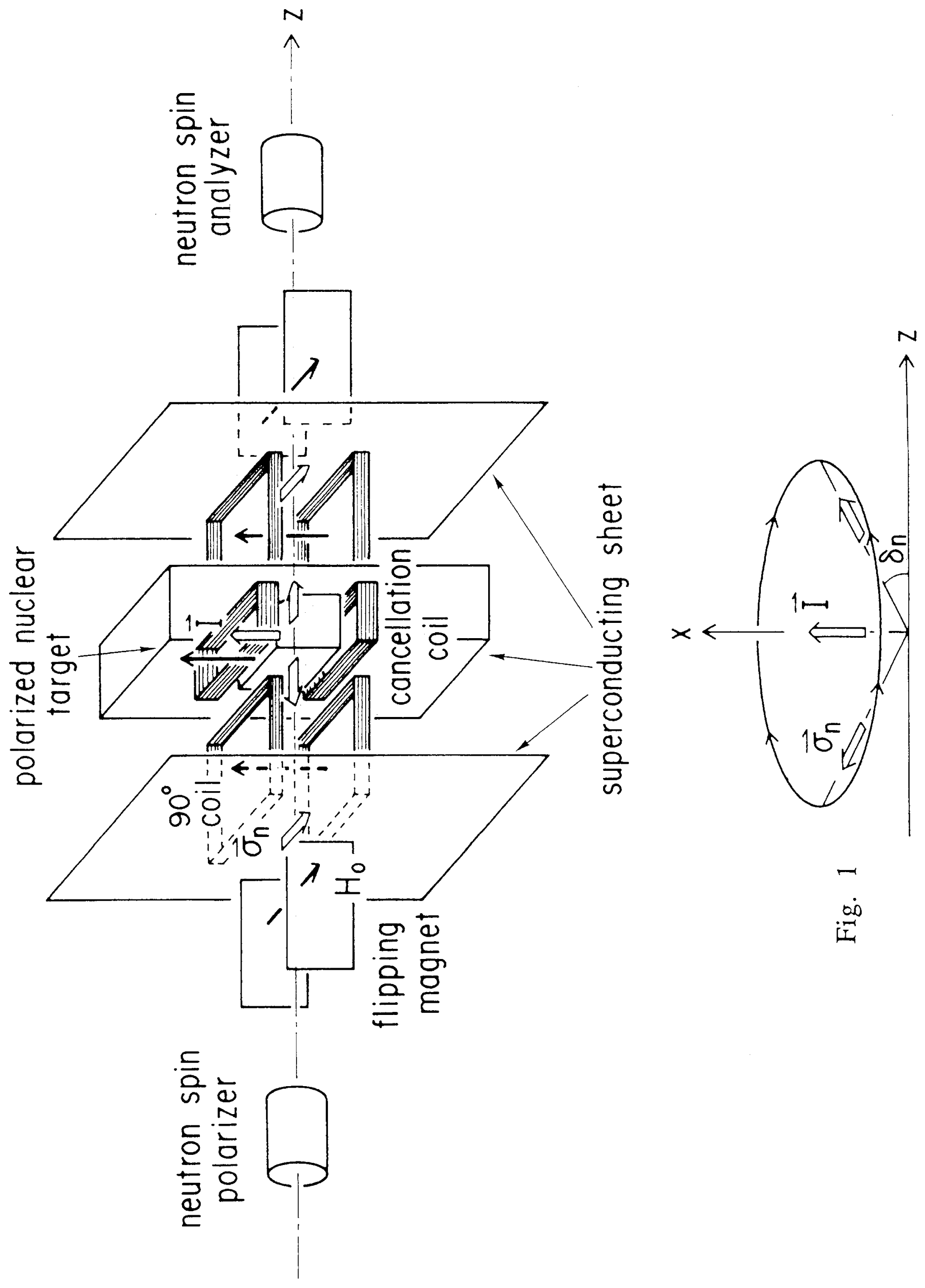


